\title{
Apoptotic cell clearance in chronic inflammation of lateral neck cysts
}

\author{
Wieslaw Dobros $\cdot$ Karolina Burda $\cdot$ \\ Krzysztof Guzik · Joanna Koziel $\cdot$ Jan Potempa
}

Received: 30 March 2011 / Accepted: 29 June 2011/Published online: 14 July 2011

(C) The Author(s) 2011. This article is published with open access at Springerlink.com

\begin{abstract}
The mechanism driving accumulation of large numbers of apoptotic and necrotic neutrophils in inflamed lateral neck cysts (LNC), in the absence of infection, remains obscure. The cellular content of cysts obtained from 17 patients was co-cultured with human macrophages. Phagocytosis levels of cyst-derived neutrophils were determined and compared to the uptake of spontaneously apoptotic neutrophils. Simultaneously, the expression of cytokines in macrophages exposed to cyst contents was measured. In comparison to spontaneously apoptotic neutrophils, the phagocytosis of LNC-derived neutrophils by macrophages was inefficient. An inverse correlation between neutrophil content in LNC and their uptake was observed. Macrophages co-cultured with cyst contents responded with variable expression of IL-6, TNF$\alpha$ and IL-10. The hindered clearance of apoptotic neutrophils in LNC may lead to secondary necrosis of these cells
\end{abstract}

\section{W. Dobros}

Department of Otolaryngology, Regional Hospital,

ul. Lwowska 178A, 33-100 Tarnow, Poland

K. Burda · J. Koziel $(\bowtie) \cdot$ J. Potempa

Department of Microbiology, Faculty of Biochemistry,

Biophysics and Biotechnology, Jagiellonian University,

ul. Gronostajowa 7, 30-387 Kraków, Poland

e-mail: joanna.koziel@uj.eu.pl

K. Guzik

Department of Immunology, Faculty of Biochemistry, Biophysics and Biotechnology, Jagiellonian University, ul. Gronostajowa 7, 30-387 Kraków, Poland

\section{J. Potempa}

Oral Health and Systemic Research Facility, School of Dentistry,

University of Louisville, Louisville, KY 40202, USA and stimulation of the inflammatory reaction. Together with local production of anti-inflammatory cytokines, this may fuel chronic inflammation in the cysts.

Keywords Lateral neck cysts · Branchial cysts · Phagocytosis · Inflammation · Apoptosis · Neutrophils

\section{Introduction}

The etiology of lateral neck cysts (LNC) is still ambiguous [1]. Although a growing number of studies indicate that cysts develop from congenital remnants of bronchial arches [2-5], others suggest that LNCs originate from cystic transformation of cervical lymph nodes [6]. Regardless of the etiology, all LNC are morphologically epithelium-lined sacks filled up with watery fluid and mucoid or gelatinous material [3]. Symptomatic enlargement of LNCs, apparently the result of immunologic stimulation, occurs most often between the second and forth decades of life. To avoid future complications the first choice of treatment is surgical excision; however, some patients delay resection until the growing discomfort and/or pain forces them to seek surgical treatment. Interestingly, although LNC infection is assumed to be the main cause of LNC enlargement, the bacteriological examination of cyst content commonly yields negative results. Indeed, even in the presence of clear symptoms, such as local inflammation, $96.2 \%$ of cyst contents are sterile [7]. At this stage, large numbers of acute inflammatory cells [5], including neutrophils and macrophages, are found in LNC content [8]. Recently, it was shown that neutrophils found in thick, yellowish pus-like fluid aspirated from inflamed LNCs are either apoptotic or necrotic, and at different stages of degeneration [9]. 
In healthy tissues, apoptotic neutrophils are specifically and efficiently removed by bystander professional phagocytes, predominantly macrophages, and non-professional phagocytes [10]. This prevents disintegration of dying neutrophils and release of their tissue-damaging proinflammatory content. In infectious inflammatory sites, uptake of apoptotic neutrophils by macrophages changes the phenotype of these latter cells from pro- to antiinflammatory [11]. Thus, the release of inflammationquenching cytokines by macrophages, which engulf apoptotic neutrophils, contributes to resolution of the inflammatory reaction and healing process [11]. The accumulation of large numbers of apoptotic and necrotic neutrophils in LNCs [9] suggests that the process of recognition and clearance of dying cells is malfunctioning. It can be assumed that this will lead to eventual disintegration of neutrophils and release of their cytoplasmic content, which fuels inflammation in the local environment of LNCs. To verify this hypothesis, in this study we have compared the phagocytosis of LNC-derived neutrophils with peripheral blood-derived apoptotic neutrophils from healthy donors. In addition, the profile of cytokines produced by macrophages upon phagocytosis of LNC-derived neutrophils was determined.

\section{Materials and methods}

\section{Lateral neck cyst fluid samples}

This study, approved by the Regional Medical Chamber in Tarnow (permit number 4/0177/2001), was performed on sterile LNC fluid obtained from 17 patients diagnosed with branchial cysts and subjected to surgery in the Department of Otolaryngology at the Regional Hospital in Tarnow, Poland, from 2001 to 2003. No signs of ongoing, generalized or local inflammation was detected in surgically treated patients. Some patients, however, suffered from previous incidences of local inflammation of the cyst, which were treated with antibiotics. Specimens of cyst fluidic matter were taken aseptically during surgery for bacteriological examination and analysis of cellular content. Patients were monitored for more than 3 years. Recurrence of cysts was not observed in this period. This means that the cyst was removed radically and they were not connected with the pharyngeal mucosa.

PBMC isolation and macrophages culture

Peripheral blood mononuclear cells (PBMCs) were obtained from the blood of healthy donors using a lymphocyte separation medium (LSM; PAA, Germany) density gradient [12]. De-identified blood was obtained from the
Red Cross (RC), Krakow, Poland, in compliance with confidentiality assurances of human subjects. Thus, the research protocol presented here adheres to the appropriate exclusions for human subjects' approval. PBMCs were seeded at a concentration of $3 \times 10^{6}$ cells per well in 24-well plates, and cultured in RPMI1640 medium (PAA, Germany) supplemented with $10 \%$ heat-inactivated autologous human plasma, $2 \mathrm{mM} \mathrm{L}$-glutamine and $50 \mu \mathrm{g} / \mathrm{mL}$ gentamicin (Sigma, St Louis, MO, USA) in a humidified atmosphere of $5 \% \mathrm{CO}_{2}$. After $24 \mathrm{~h}$, non-adherent cells were removed and adherent monocytes were differentiated into macrophages for 7-10 days, with fresh medium changes every second day. hMDM phenotype was evaluated by flow cytometry analysis of detached cells immunofluorescently stained for CD14 (DakoCytomation, Glostrup, Denmark), CD16 (DakoCytomation, Glostrup, Denmark), CD11b (Becton-Dickinson, San Jose CA, USA) and CD209 (Becton-Dickinson, San Jose CA, USA). This routine procedure used in our laboratory yields at least $90 \%$ of cells positive for the first three antigens with less than $1 \%$ staining with anti-CD209 antibodies.

\section{Polymorphonuclear cell isolation}

Cells in peripheral blood were subjected to sedimentation in $1 \%$ polyvinyl alcohol solution (Merck, Darmstadt, Germany) for $20 \mathrm{~min}$ at room temperature. Neutrophils were collected from the upper layer of cells and lysed with distilled water for $20 \mathrm{~s}$ to remove contaminating erythrocytes. After isolation, neutrophils were cultured in RPMI1640 medium (PAA, Germany) supplemented with $5 \%$ FBS for $24 \mathrm{~h}$. Such treatment induced spontaneous apoptosis of more than $90 \%$ neutrophils as evaluated by annexin V/PI staining via FACS [13].

Phagocytosis of neutrophils—an elastase activity assay

The assay was performed as described in detail by Smagur et al. [13]. Briefly, a suspension of $1.5 \times 10^{6}$ apoptotic neutrophils in media containing 5\% fetal bovine serum (FBS, PAA, Germany) was added to hMDMs at an hMDM:neutrophils ratio of 1:5 in a 24-well culture plate. Simultaneously, $100 \mu \mathrm{L}$ of LNC fluid from ten patients was added to the hMDMs. Cells were incubated for $2 \mathrm{~h}$ at $37^{\circ} \mathrm{C}$ in a humidified atmosphere containing $5 \% \mathrm{CO}_{2}$, and monolayers were washed vigorously with ice-cold PBS to remove unphagocytosed neutrophils. After washing, the macrophage monolayer was lysed with $0.1 \%$ cetyltrimethylammonium bromide for $15 \mathrm{~min}$ at $37^{\circ} \mathrm{C}$, and $100 \mu \mathrm{L}$ of lysates were transferred, in quadruplicate, to a 96-well plate, followed by the addition of $100 \mu \mathrm{L}$ of an elastase substrate ( $N$-methoxysuccinyl-Ala-Ala-Pro-Val- $p$ nitroanilide) at a final concentration of $1 \mathrm{mM}$, in Tris- $\mathrm{HCl}$ 
buffer, $\mathrm{pH}$ 7.5. Elastase activity ( $V_{\max }$ of enzymatic reaction at $37^{\circ} \mathrm{C}$ ), equivalent to the released $p$-nitroanilide, was recorded as an increase in absorbance at $405 \mathrm{~nm}$ for 30 min using a microplate reader (Molecular Devices, Sunnyvale, CA, USA). The percentage of engulfed neutrophils was calculated based on neutrophil elastase activity levels detected in cell lysates after phagocytosis. The activity in lysates of equivalent numbers of cells, directly treated with detergent, was taken as $100 \%$.

Determination of mRNA expression for IL-6, IL-10 and TNF- $\alpha$ in macrophages

Total RNA was isolated from hMDMs, after exposure to cyst samples, using the TRIZOL method (GibcoBRL, Grand Island, NY, USA) according to the manufacturer's instruction. Following precipitation, RNA was resuspended in RNase-free DEPC-treated water and treated with DNAse (Qiagen, Hilden, Germany) solution for $30 \mathrm{~min}$. Following enzyme inactivation by heating for $5 \mathrm{~min}$ at $75^{\circ} \mathrm{C}$, RNA concentration was determined by absorbance at a wavelength of $260 \mathrm{~nm}$. cDNA was synthesized from total cellular RNA $(5 \mu \mathrm{g})$ using $200 \mathrm{U}$ SuperScript TM II reverse transcriptase (GibcoBRL, Grand Island, NY, USA) and $0.5 \mu \mathrm{g} / \mu \mathrm{l}$ oligo (dT) primers (GibcoBRL, Paisley, UK). After reverse transcription, enzyme activity was destroyed by heating and the cDNA stored at $-20^{\circ} \mathrm{C}$ until ready for use in real-time PCR. The expression of cytokines was determined using specific primers (see Table 1). Concomitantly, amplification of the control gene $\beta$-actin (Genset Oligos, Paris, France) was performed on the same samples to verify RNA integrity. Each PCR-product $(15 \mu \mathrm{L})$ was electrophoresed on a $2 \%$ agarose gel stained with ethidium bromide and then visualized with UV light. The correct size of predicted PCR products was confirmed using a 100-bp ladder (Gibco BRL/Life Technologies, Eggenstein, Germany) as a standard marker.

\section{Results}

The contents of lateral neck cysts was aspirated using a thin needle and subjected to macroscopic examination. With the exception of specimen no. 8, where the cyst matter was watery, clear and colorless, the LNC fluid was pale-brown or yellow-orange colored, dense and viscous. Next, the specimens were plated for bacteriological examination; however, no bacterial growth was detected, indicating that the collected cyst fluid was sterile (data not shown).

Based on previous findings [9] that LNC content is rich in apoptotic neutrophils, we designed experiments to test the susceptibility of these cells to engulfment by human macrophages. To this end, we first determined neutrophil elastase activity in all samples as a measure of neutrophil content; with significant activity found in 10 out of 17 samples. Next, using apoptotic neutrophils as a standard, we estimated that the cell content of our ten positive samples was in the range of $0.21 \times 10^{6}$ $5.62 \times 10^{6}$ per $\mathrm{mL}$ of analyzed fluid (Table 2 ). To determine the phagocytosis of neutrophils in these ten samples by macrophages, the cellular content of cyst fluids were cocultured with hMDMs, and the level of phagocytosis estimated as described above. In vitro prepared, spontaneously apoptotic neutrophils were taken up by hMDMs far more efficiently than the neutrophils present in LNC fluid. Interestingly, an inverse correlation between neutrophil content in LNC fluid samples was observed, since the uptake of neutrophils from samples with high content of these cells [sample 10,11,12, and 15, neutrophils content $>2 \times 10^{6}$ per mL (Table 2)] was at very low levels (below 3\%). In contrast, more than $10 \%$ of apoptotic neutrophils were phagocytosed by macrophages from four out of the six samples with a neutrophil content ranging from $0.21 \times 10^{6}$ to $0.86 \times 10^{6}$ per mL (Fig. 1). Regardless of these differences, it is clear that phagocytosis of neutrophils from cysts is very inefficient by comparison to clearance of apoptotic cells.

Table 1 Primers used in PCR amplification

\begin{tabular}{|c|c|c|c|c|}
\hline Primer & & Sequence & Product (bp) & Annealing temperature $\left({ }^{\circ} \mathrm{C}\right)$ \\
\hline \multirow[t]{2}{*}{$\beta$-actin } & AS & $5^{\prime}$ GGGTACATGGTGGTGCCG $3^{\prime}$ & 307 & 55 \\
\hline & $\mathrm{S}$ & $5^{\prime}$ AGCGGGAAATCGTGCGTG 3' & & \\
\hline \multirow[t]{2}{*}{ IL-6 } & AS & $5^{\prime}$ GCTGCGCAGAATGAGATGAGTTGTC $3^{\prime}$ & 237 & 55 \\
\hline & $\mathrm{S}$ & $5^{\prime}$ CTTTTGGAGTTTGAGGTATACCTAG $3^{\prime}$ & & \\
\hline \multirow[t]{2}{*}{ TNF- $\alpha$} & AS & $5^{\prime}$ GCAATGATCCCAAAGTAGACCTGCCC $3^{\prime}$ & 702 & 60 \\
\hline & $\mathrm{S}$ & $5^{\prime}$ ATGAGCACTGAAAGCATGATC $3^{\prime}$ & & \\
\hline \multirow[t]{2}{*}{ IL-10 } & AS & $5^{\prime}$ CTGAGAACCAAGACCCAGACATCAAGG $3^{\prime}$ & 328 & 60 \\
\hline & $\mathrm{S}$ & $5^{\prime}$ GTCAGCTATCCCAGAGCCCCAGATCCG $3^{\prime}$ & & \\
\hline
\end{tabular}


Table 2 Neutrophil elastase activity of apoptotic neutrophils as a measure of the numbers of neutrophils in LNC content

\begin{tabular}{lcl}
\hline Specimen no. & NE activity $(\mathrm{mOD} / \mathrm{min})$ & PMN $/ \mathrm{mL}$ \\
\hline 1 & 0 & N.d. \\
2 & 12 & $0.85 \times 10^{6}$ \\
3 & 12.2 & $0.86 \times 10^{6}$ \\
4 & 7 & $0.49 \times 10^{6}$ \\
5 & 8 & $0.56 \times 10^{6}$ \\
6 & 6.5 & $0.46 \times 10^{6}$ \\
7 & 0 & N.d. \\
8 & 0 & N.d. \\
9 & 0 & N.d. \\
10 & 29 & $2.06 \times 10^{6}$ \\
11 & 53 & $3.77 \times 10^{6}$ \\
12 & 36 & $2.56 \times 10^{6}$ \\
13 & 0 & N.d. \\
14 & 0 & N.d. \\
15 & 79 & $5.62 \times 10^{6}$ \\
16 & 0 & N.d. \\
17 & 3 & $0.21 \times 10^{6}$ \\
\hline
\end{tabular}

Activity was determined with a chromogenic substrate (MeO-SucAla-Ala-Pro-Val-pNA) specific for neutrophil elastase (NE). One hundred microliters of cyst contents (1-17) was solubilized with a cetyltrimethylammonium bromide-containing buffer and the NE activity was determined as a time-dependent increase of absorbance at $405 \mathrm{~nm}$. Results are represented in $\mathrm{mOD} / \mathrm{min}$. In parallel, the elastase activity in known numbers of spontaneously apoptotic neutrophils was determined and used to convert $\mathrm{mOD} / \mathrm{min}$ into neutrophils concentration (cells/mL) in LNC content

$N . d$. the lack of elastase activity does not exclude the absence of PMNs

LNC content may contain blood-derived protease inhibitors which may quench NE activity

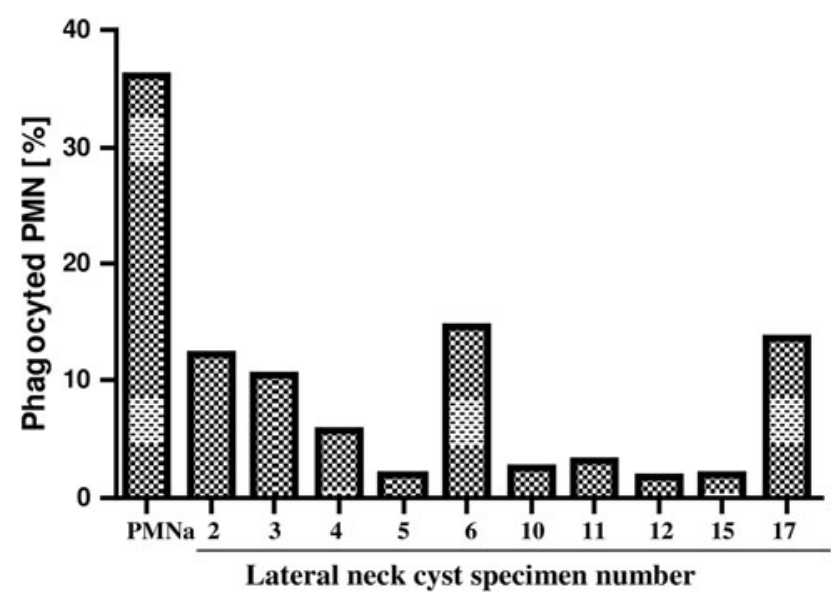

Fig. 1 A comparison of efficiency of phagocytosis of spontaneously apoptotic neutrophils and neutrophils derived from LNC content by human primary macrophages
The phagocytosis of apoptotic cells is crucial for maintaining homeostasis in tissues, and has a strong effect on the immunological system. Upon uptake of apoptotic cells, macrophages are directed toward anti-inflammatory responses manifested by changes in the profile of produced cytokines [11]. Therefore, we have investigated the effect of macrophage exposure to LNC cellular content on the expression of pro- (IL- 6 and TNF- $\alpha$ ) and anti-inflamatory (IL-10) cytokines. As a control, bacterial endotoxin (LPS from $E$. coli) was used as a factor to strongly stimulate a proinflammatory response (Fig. 2). In comparison to LPS, macrophage response to cyst contents was weaker, especially for IL- 6 and TNF- $\alpha$ transcription. Also, there was a clear difference in response of hMDMs to stimulation with individual samples; for example, the IL-6 transcript was detected in all cases, but at different levels. The highest accumulation of IL- 6 mRNA was observed in seven samples $(1,3,4,5,7,11$ and 12) (Fig. 2a), while in contrast the expression of TNF- $\alpha$ was detected in macrophages treated with only six samples (3, 4, 5, 6, 13 and 16) (Fig. 2b). Finally, mRNA for IL-10 was detected in macrophages exposed to 12 out of 17 tested LNC specimens. Interestingly, for five of these samples $(3,4,5,6$ and 17), the response was at a level equivalent to that induced by LPS (Fig. 2c). No correlation could be found between neutrophil content and their susceptibility to engulfment by macrophages, and cytokine response. The results presented reveal that only the fluid from individual cysts can induce variable expression of IL-6, TNF- $\alpha$ and IL-10 in macrophages, which may bias the immune response in LNCs toward development and maintenance of a chronic inflammatory reaction.

\section{Discussion}

Little is known about the role of inflammatory cells in the pathology of LNCs. To bridge this gap, we describe here a possible new mechanism contributing to chronic inflammatory reactions through the dysfunction of apoptotic cell clearance in such cysts. We have shown that neutrophils extracted from cysts were engulfed by macrophages significantly less efficiently than apoptotic neutrophils. Reproducibly, about $30 \%$ of spontaneously apoptotic PMNs co-cultured with macrophages were phagocytosed. This number is in stark contrast to the uptake of cystderived neutrophils, which was in the range of $2-14.3 \%$. This finding may explain the high numbers of apoptotic and necrotic cells in the LNC content reported previously [9]. The hindered local clearance of neutrophils in cysts is most likely due to both disabling of "eat-me" signals on the surface of apoptotic neutrophils, and proteolytic inactivation of receptors recognizing "eat-me" signals on phagocytic cells by neutrophil elastase. Such a mechanism 
Fig. 2 Determination of cytokine mRNA by PCR in macrophages stimulated by LNC content. $C$ control cells, LPS macrophages stimulated with LPS (1 ng/ml), 1-17 macrophages stimulated with the LNC content aspired from patients numbered from 1 to 17
Lateral neck cyst specimen number

A

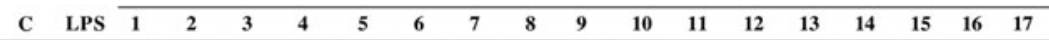

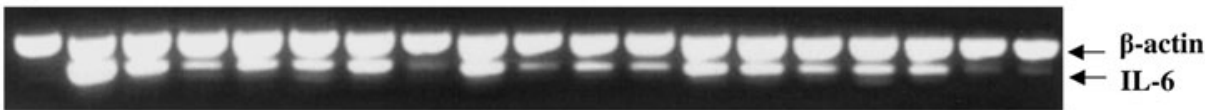

B

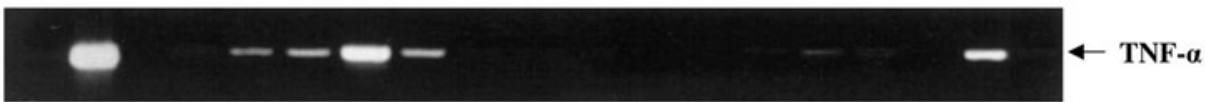

C

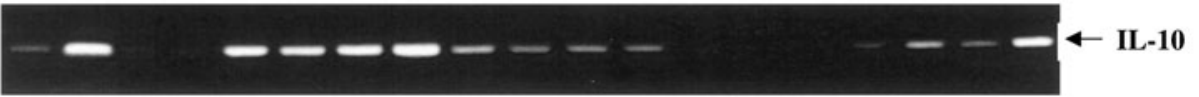

of disturbed clearance of apoptotic neutrophils has previously been described for cystic fibrosis [14]. Significantly, this mechanism potentially explains the inverse correlation between the number of neutrophils in cyst content, measured by the elastase activity, and the efficiency of neutrophil uptake by macrophages, described in this report.

The phagocytosis of apoptotic cells by macrophages plays a very important role in regulation of the immune system [15]. Firstly, it prevents leakage of pro-inflammatory factors from dying cells and, secondly, this process can induce an antiinflammatory phenotype in macrophages, manifested by a qualitative change in cytokine production. Here, we have shown that LNC content induces a strong, but mixed pro- and then anti-inflammatory response, manifested by IL- 6 , TNF- $\alpha$ and IL-10 production. Taking into account the sterile character of LNC content, this response is most likely driven by endogenous factors, such as enzymes and peptides released from dying cells. In contrast to anti-inflammatory IL-10, proinflammatory TNF- $\alpha$ was induced much less frequently (by 12 vs. 5 samples out of the 17 tested). Such a pattern of expression of these two cytokines, together with the prevalent production of IL- 6 by macrophages exposed to LNC content, may have important pathological consequences. Acting synergistically with IL- $1 \beta$ and TNF- $\alpha$, IL- 6 can contribute to acute inflammation, while at the same time prolonged activity of this cytokine can silence an inflammatory reaction [16]. In this context, the biological activities of TNF- $\alpha$ and IL- 6 are antagonistic: instead of helping to resolve inflammation they may contribute to maintaining an inflammatory reaction. This tendency can be further strengthened by anti-inflammatory IL-10, which inhibits IFN- $\gamma$, IL- $1 \beta$ and TNF- $\alpha$ production and antigen presentation [17]. Therefore, macrophage response to LNC content has the potential to establish a chronic inflammatory state in cysts.

\section{Conclusion}

Taken together, based on our findings, we hypothesize that the hindered clearance of apoptotic neutrophils in LNC matter leads to secondary necrosis of these cells, and stimulation of the inflammatory reaction. Simultaneously, the local production of significant levels of anti-inflammatory cytokines by stimulated macrophages prevents the resolution of the inflammatory state. This leads to selfpropelled chronic inflammation in the cysts, which can only be resolved by surgery.

Acknowledgments This work was supported by the Jagiellonian University (statutory funds DS/9/WBBiB). The Faculty of Biochemistry, Biophysics and Biotechnology of the Jagiellonian University is a beneficiary of the structural funds from the European Union (Grant No. POIG.02.01.00-12-064/08_ “Molecular biotechnology for health").

Conflict of interest The authors declare that they have no conflict of interest.

Open Access This article is distributed under the terms of the Creative Commons Attribution Noncommercial License which permits any noncommercial use, distribution, and reproduction in any medium, provided the original author(s) and source are credited.

\section{References}

1. Mandell DL (2000) Head and neck anomalies related to the branchial apparatus. Otolaryngol Clin North Am 33:1309-1332

2. Bocciolini C, Dall'Olio D, Cunsolo E, Latini G, Gradoni P, Laudadio P (2006) Cervical bronchogenic cyst: asymptomatic neck mass in an adult male. Acta Otolaryngol 126:553-556

3. Regauer S, Gogg-Kamerer M, Braun H, Beham A (1997) Lateral neck cysts - the branchial theory revisited. A critical review and clinicopathological study of 97 cases with special emphasis on cytokeratin expression. APMIS 105:623-630

4. Newkirk KA, Tassler AB, Krowiak EJ, Deeb ZE (2004) Bronchogenic cysts of the neck in adults. Ann Otol Rhinol Laryngol 113:691-695

5. Thomaidis V, Seretis K, Tamiolakis D, Papadopoulos N, Tsamis I (2006) Branchial cysts. A report of 4 cases. Acta Dermatovenerol Alp Panonica Adriat 15:85-89

6. Golledge J, Ellis H (1994) The aetiology of lateral cervical (branchial) cysts: past and present theories. J Laryngol Otol 108:653-659

7. Makowska W, Waloryszek B, Rudnicka J (1998) Badanie bakteriologiczne treści z torbieli szyi. Otolaryng Pol 52:527-529 
8. Glosser JW, Pires CA, Feinberg SE (2003) Branchial cleft or cervical lymphoepithelial cysts: etiology and management. J Am Dent Assoc 134:81-86

9. Dobros W, Burda K, Pacura B, Potempa J, Guzik K (2010) The presence of apoptotic neutrophils in contents of neck lateral cysts. Przeglad Lek 67:357-359

10. Fadeel B, Xue D, Kagan V (2010) Programmed cell clearance: molecular regulation of the elimination of apoptotic cell corpses and its role in the resolution of inflammation. Biochem Biophys Res Commun 396:7-10

11. Huynh ML, Fadok VA, Henson PM (2002) Phosphatidylserinedependent ingestion of apoptotic cells promotes TGF-beta1 secretion and the resolution of inflammation. J Clin Invest 109:41-50

12. Koziel J, Maciag-Gudowska A, Mikolajczyk T et al (2009) Phagocytosis of Staphylococcus aureus by macrophages exerts cytoprotective effects manifested by the upregulation of antiapoptotic factors. PLoS One 4(4):e5210
13. Smagur J, Guzik K, Bzowska M et al (2009) Staphylococcal cysteine protease staphopain $\mathrm{B}(\mathrm{SspB})$ induces rapid engulfment of human neutrophils and monocytes by macrophages. Biol Chem 390:361-371

14. Vandivier RW, Fadok VA, Hoffmann PR et al (2002) Elastasemediated phosphatidylserine receptor cleavage impairs apoptotic cell clearance in cystic fibrosis and bronchiectasis. J Clin Invest 109:661-670

15. Savill J, Dransfield I, Gregory C, Haslett C (2002) A blast from the past: clearance of apoptotic cells regulates immune responses. Nat Rev Immunol 2:965-975

16. Diehl S, Rincón M (2002) The two faces of IL-6 on Th1/Th2 differentiation. Mol Immunol 39:531-536

17. Couper KN, Blount DG, Riley EM (2008) IL-10: the master regulator of immunity to infection. J Immunol 180:5771-5777 